\title{
Co-integração entre os mercados spot e futuro: evidências dos mercados de boi gordo e soja
}

Kleber Giovelli Abitante ${ }^{1}$

Resumo: Uma das medidas de eficiência dos mercados futuros refere-se à sua ligação com o mercado spot (mercado à vista). Este trabalho teve por objetivo verificar se existe uma ligação estatística entre o mercado spot e futuro de boi gordo negociado na Bolsa de Mercadorias e Futuros (BM\&F) e entre o mercado spot e futuro de soja negociados na BM\&F e na Chicago Board of Trade (СВOT). Além disso, procedeu-se também o cálculo de um indicador de eficiência para o contrato futuro de boi gordo da BM\&F. Para o boi gordo, os dados utilizados foram as séries de preços futuros dos contratos com vencimento nos meses de janeiro a novembro/2005 e para os contratos de soja os vencimentos escolhidos foram de março a setembro/2005, além de novembro/2005. No caso do mercado de boi gordo, foram encontradas evidências de co-integração entre os preços spot e os contratos com vencimento em março, abril, setembro, outubro e novembro/2005. No caso do contrato de soja da BM\&F, a co-integração foi detectada nos vencimentos de abril, maio e novembro/2005. O contrato de soja da CBOT apresentou co-integração nos vencimentos de janeiro, março, setembro e novembro/2005. O indicador de eficiência do contrato futuro de boi gordo mostrou-se elevado, indicando que o mesmo pode auxiliar na descoberta de preço desta commoditie.

Palavras-chaves: Co-integração; Mercado futuro; Boi gordo; Soja.

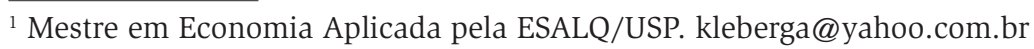


Classificação JEL: C32, Q1, Q11

\begin{abstract}
One of the measures of future markets' efficiency is its linkage with the spot market. The objective of this paper is to verify the existence of a statistical linkage between spot market and the Brazilian Mercantile \& Future Exchange (BM\&F) live cattle future market and between spot market and the BM\&F and Chicago Board of Trade (CBOT) soybean future market. In addition, an efficiency indicator for the BM\&F live cattle future market was estimated. With regard to live cattle, the daily time series used was price of the future contracts with maturity month between January/05 and November/05 and for soybean, the price of the future contracts used was with maturity between March 2005 until September 2005 and November 2005. Concerning live cattle, evidences of co-integration on contracts with maturity month on March, April, September, October and November of 2005 were found. As for the BM\&F soybean future contract, co-integration was detected for contracts with maturity at April, May and November, 2005. Co-integration evidence was identified for the CBOT soybean future with maturity at January, March, September and November of 2005. The efficiency indicator for live cattle future contract was higher, indicating that this market may be useful for price discovery of live cattle.
\end{abstract}

Keywords: Cointegration; Futures markets; Soybean; Live cattle.

JEL Classification: C32, Q1, Q11

\title{
1. Introdução
}

A atividade agropecuária apresenta um grande número de riscos inerentes ao seu perfil, como difícil previsibilidade de preço e de produção, os quais, por sua vez, são decorrentes de fatores fora da alçada de controle por parte do produtor, como variações na oferta e demanda, intempéries climáticas e enfermidades durante a produção. Uma das principais funções do mercado futuro inclui a estabilização da variável preço na equação de lucro, por meio da realização da descoberta de preço. A descoberta de preço em mercados futuros pode ser definida 
como o uso de preços futuros para determinar expectativas de preço para o mercado spot (mercado do produto acabado para venda e com pronta disposição de entrega), fornecendo ao produtor a oportunidade de assegurar uma dada cotação em troca do risco de oscilação da base, a qual apresenta menor volatilidade. Esta função de descoberta de preço pode ser mais eficientemente realizada pelo mercado futuro quanto maior o grau em que este se encontra correlacionado ou ligado ao mercado físico (WANG \& YAU, 1994).

Grande parte da literatura elaborada antes dos anos de 1980 argumenta que existe uma relação funcional (integração) entre preços spot e preços futuros para commodities estocáveis, mas, no entanto, esta relação não existe para as commodities não estocáveis (como boi gordo e suínos, por exemplo). A partir do início da década de 1990, quando se passou a utilizar a análise de co-integração para investigar a descoberta de preço, os resultados foram diferenciados. Alguns trabalhos argumentam que a ligação entre o mercado spot e o mercado futuro depende da armazenabilidade do ativo. Outro grupo de trabalhos sugere que a ausência de integração para commodities não estocáveis ocorreria em virtude de erro de especificação do problema, ou seja, a exclusão de possíveis elementos não estacionários na co-integração. Alguns autores encontraram evidências diversificadas de integração entre commodities estocáveis, porém, nenhuma integração para commodities não estocáveis (YANG et al., 2001).

O objetivo deste trabalho é verificar se existe uma ligação estatística de longo-prazo entre o mercado físico e o mercado futuro das commodities boi gordo e soja, no âmbito do Brasil, com o intuito de se verificar se o mercado futuro pode fornecer informações que auxiliem os produtores no processo de descoberta de preço, e também analisar a eficiência com que o mercado futuro pode predizer o preço spot. No caso do boi gordo, o mercado futuro considerado refere-se ao contrato de boi gordo da Bolsa de Mercadorias \& Futuros (BM\&F), e no caso da soja foram analisados os contratos futuros de soja da BM\&F e da Chicago Board of Trade (СВOT). Em virtude da não estacionariedade das variáveis em questão, será utilizada a análise de co-integração proposta por Johansen (1988) e Johansen \& Juselius (1990) e exposta em Lai \& Lai (1991).

Este trabalho possui, além desta introdução, outras cinco seções. Na 
seção 2 são apresentadas características dos mercados físico e futuro das commodities boi gordo e soja. Na seção 3 apresenta-se a metodologia empregada na consecução do objetivo proposto. Na seção 4 são expostas as características da amostra de dados utilizada. Na seção 5 os resultados são apresentados e discutidos. Finalmente, na seção 6 constam as conclusões do trabalho, bem como as limitações do mesmo e propostas de pesquisas futuras.

\section{Os mercados futuro e físico de boi gordo e soja no Brasil}

\subsection{Mercados de boi gordo}

O Brasil possui uma das maiores produção de gado bovino do mundo. O rebanho bovino brasileiro, em 2004, foi de 170,1 milhões de cabeças, o segundo maior do mundo, representando $16,7 \%$ do rebanho mundial, atrás apenas da Índia, que possuía 330,2 milhões de cabeças no mesmo ano. Deste total, 117,5 milhões de cabeças são bovinos de corte, aproximadamente $69 \%$ do rebanho total (INSTITUTO FNP, 2005).

A produção brasileira de carne bovina, em 2004, foi de 8,4 milhões de toneladas de equivalente-carcaça (EC), o que representa $16,4 \%$ da produção mundial, sendo o segundo maior produtor, atrás apenas dos EUA, que produziram 11,2 milhões de toneladas no mesmo período. As exportações de carne bovina industrializada foram da ordem de $171 \mathrm{mil}$ toneladas em 2004, volume $27,4 \%$ maior que em 2001, e as exportações de carne in natura foram de 925 mil toneladas, montante $151,2 \%$ maior que em 2001. Em 2004, o Brasil foi o maior exportador de carne bovina, com cerca de 1,6 milhões de toneladas de EC exportadas (cerca de 25,4\% das exportações mundiais), seguido da Austrália, com 1,3 milhões de toneladas e a Índia com 540 mil toneladas (INSTITUTO FNP, 2005).

$\mathrm{O}$ contrato futuro de boi gordo negociado na BM\&F tem o seu formato atual desde $09 / 2000$. O objeto do contrato é o boi gordo acabado para abate, com peso entre 450 e 550 quilos e idade máxima de 42 meses. Cada contrato é cotado em reais por arroba e possui tamanho padrão de 330 arrobas, havendo vencimento para todos os meses do ano. A data de vencimento é o último dia útil do mês, e o ponto de entrega (e, consequentemente, de formação de preço), está localizado 
no município de Araçatuba (SP). O valor de liquidação do contrato é calculado como a média ponderada dos cinco últimos indicadores ESALQ/BM\&F da arroba do boi gordo. Em 2005, este contrato futuro foi o segundo contrato agropecuário com maior liquidez na BM\&F - atrás apenas no contrato futuro de café arábica - sendo que em novembro de 2005 foram negociados 22,6 mil contratos, montante $34,7 \%$ superior ao mesmo mês de 2004 . No fechamento de novembro de 2005 , havia 8,6 mil contratos em aberto, registrando uma queda de 30,9\% em relação à novembro de 2004. O volume de contratos futuros de boi gordo negociados de janeiro a novembro de 2005 foi de 289,8 mil contratos, montante este $44,2 \%$ superior ao volume negociado no mesmo período de 2004 (BM\&F, 2005a; BM\&F, 2005b).

\subsection{Mercado de soja}

A produção de soja e seus derivados é um das maiores cadeias agroindustriais do Brasil. A produção brasileira de soja em grãos foi de aproximadamente 51,0 milhões de toneladas métricas na safra de $2004 / 05$, cerca de $2,6 \%$ superior ao montante da safra 2003/04, sendo o segundo maior produtor, atrás dos EUA, que obtiveram 78,2 milhões de toneladas na referida safra. A área plantada na safra 2004/05 foi de 23,3 milhões de hectares, àrea 9,0\% superior à da safra anterior. Desta forma, a taxa de produtividade em 2004/05 foi de 2,19 kg/ha, valor $5,8 \%$ inferior à taxa da safra anterior (CONAB, 2005).

As exportações de soja em grãos do Brasil foram de 13,3 milhões de toneladas de janeiro a julho de 2005, representando cerca de 19,0\% das exportações mundiais. Os principais importadores da soja em grãos brasileira foram China, Países Baixos, Alemanha e Espanha (CONAB, 2005; FNP CONSULTORIA \& AGROINFORMATIVOS, 2005).

O contrato futuro de soja em grãos a granel da BM\&F tem como objeto de negociação a soja para exportação, sendo que os níveis de impurezas, umidade e outras características do produto são padronizados. O contrato é cotado em dólares dos EUA por saca de $60 \mathrm{~kg}$, sendo que cada contrato refere-se a 27 toneladas métricas ou 450 sacas de $60 \mathrm{~kg}$. Os meses de vencimento são: março, abril, maio, junho, julho, agosto, setembro e novembro. A data de vencimento refere-se ao nono dia útil 
anterior ao primeiro dia do mês de vencimento e o ponto de entrega do produto é uma unidade de carregamento de navios no corredor de exportação de Paranaguá (PR) (BM\&F, 2005c).

Em 2005, o contrato futuro de soja da BM\&F foi um dos contratos agropecuários de menor liquidez da BM\&F, o que contrasta com a grande produção brasileira desta commodity. Em novembro/2005, foram negociados 3,7 mil contratos futuros de soja, montante $33,3 \%$ superior ao apurado no mesmo mês do ano anterior. Em novembro/2005, haviam 3,3 mil contratos em aberto, montante $40,7 \%$ superior ao registrado em novembro/2004. No período de janeiro a novembro/2005 foram negociados 43,2 mil contratos, aumento de $583,8 \%$ em relação ao mesmo período de 2004 (BM\&F, 2005a).

O contrato futuro de soja a granel da CBOT tem tamanho padrão de 5.000 bushels (um bushel equivale a $27,21555 \mathrm{~kg}$ ), é cotado em centavos e quartos de centavos de dólar dos EUA por bushel e tem vencimento nos meses de janeiro, março, maio, julho, agosto, setembro e novembro. O produto pode ser entregue em um dos pontos de embarque de soja nos rios Illinois ou Mississipi, entre as cidades de Chicago e St. Louis (EUA). Em 12/2005, foram negociados 1,84 milhão de contratos de soja a granel na CBOT, correspondendo a $31,9 \%$ e $4,4 \%$ dos contratos agrícolas e do total dos contratos, respectivamente, negociados no mesmo período. Em 2005 foram negociados 20,2 milhões de contratos de soja, $7,3 \%$ a mais que em 2004, o que corresponde a $26,7 \%$ dos contratos agrícolas e $2,99 \%$ e do total de contratos negociados naquela bolsa em 2005 (CBOT, 2006).

\section{Metodologia}

\subsection{Teste de co-integração}

Antes de realizar o teste de co-integração, faz-se necessário verificar se as séries são estacionárias ou possuem raiz unitária. Para verificar se a séries de preços futuros e físicos são estacionárias, utilizou-se o teste de Dickey-Fuller Aumentado, conforme procedimento seqüencial proposto por Enders (2004). A Equação (1) deve ser estimada para esse teste (ENDERS, 2004): 


$$
\Delta y_{t}=a_{0}+\gamma y_{t-1}+a_{2} t+\sum_{i=1}^{k} \beta_{i} \Delta y_{t-i}+\varepsilon_{t}
$$

A escolha do valor de $\mathrm{k}$ foi realizada por meio da avaliação dos valores de Akaike's Information Criterion (AIC) e Schwarz Bayesian Information Criterion (SBC) e também da estatística-Q de Ljung \& Box (1978). Foi testada a hipótese nula de que $\gamma=0$, contra a hipótese alternativa de que $\gamma<0$, por meio das estatísticas $\tau, \tau_{\mu}$ e $\tau_{\tau}$ de Fuller (1976), sendo testada também a significância estatística da constante e da tendência por meio das estatísticas $\phi_{1}$ e $\phi_{3}$ de Dickey e Fuller (1981). O número de raízes unitárias na série indica a sua ordem de integração, ou seja, uma série que possui uma raiz unitária é integrada de ordem 1, ou I(1).

A principal característica de variáveis co-integradas refere-se ao fato de que as suas trajetórias temporais são influenciadas por qualquer desvio de seu equilíbrio de longo prazo. Engle e Granger (1987) propõem a seguinte definição de co-integração: os componentes do vetor $y_{t}=$ $\left(y_{1 t}, y_{2 t}, \ldots, y_{n t}\right)$ são ditos serem co-integrados de ordem $d$, $b$, sendo este denotado por $y_{t} \sim \mathrm{CI}(d, b)$ se: a) todos os componentes de $y_{t}$ são integrados de ordem $d$, e; b) existe um vetor $\beta=\left(\beta_{1}, \beta_{2}, \ldots, \beta_{n}\right)$ tal que a combinação linear $\beta_{y t}=\beta_{1} y_{1 t}+\beta_{2} y_{2 t}+\ldots+\beta_{n} y_{n t}$ é integrada de ordem $(d-b)$ onde $b>0$. O vetor $\beta$ é chamado de vetor de co-integração.

Neste trabalho, utiliza-se a metodologia proposta por Johansen (1988) e Johansen \& Juselius (1990) para detectar a presença de vetores de co-integração, composta pelas seguintes etapas:

$1^{\circ}$ ) Verificar, por meio de teste de raiz unitária, se as variáveis são integradas de mesma ordem;

$2^{\circ}$ ) Para apresentar a equação a ser estimada, parte-se do seguinte modelo de Auto-Regressão Vetorial (VAR):

$$
\mathbf{y}_{t}=\boldsymbol{\mu}+\Gamma_{1} \mathbf{y}_{t-1}+\ldots+\Gamma_{p} \mathbf{y}_{t-p}+\varepsilon_{t}
$$

Após uma série de transformações algébricas, a eq. (2) pode ser escrita como:

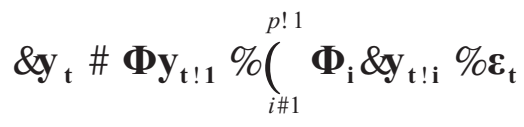

onde: $\boldsymbol{\Phi}=-\left(\mathbf{I}-\sum_{i=1}^{p} \boldsymbol{\Gamma}_{\mathbf{i}}\right)$ e $\boldsymbol{\Phi}_{\mathbf{i}} \square \square \square_{j \square \square ! 1}^{p}$ j 
O número de vetores de co-integração é igual ao rank da matriz $\mathbf{\Phi}$, obtida a partir da estimação do sistema representado pela eq. (3).

Para identificar o número de vetores de co-integração, Johansen (1988) propõe duas estatísticas:

$$
\begin{aligned}
& \lambda_{\text {trace }}(r)=-T \sum_{i=r+1}^{n} \ln \left(1-\hat{\lambda}_{i}\right) \\
& \lambda_{\max }(r)=-T \ln \left(1-\hat{\lambda}_{r+1}\right)
\end{aligned}
$$

sendo que: $\hat{\lambda_{i}}=$ os valores estimados das raízes características obtidas da matriz $\Phi$ estimada;

$T=$ número de observações utilizadas na estimação da eq. (3).

A primeira estatística (Estatística do Traço) testa a hipótese nula de que o número de vetores de co-integração distintos é menor ou igual a $r$, contra a hipótese alternativa de que ele é maior que $r$. A última estatística (Estatística do Máximo Autovalor) testa a hipótese nula de que o número de vetores de co-integração é $r$, contra a hipótese alternativa de que o número de vetores é $r+1$. Os valores críticos de $\lambda_{\text {trace }}$ e $\lambda_{\text {ma }} x$ podem ser obtidos em MacKinnon, Haug e Michelis (1999). Geralmente, adota-se apenas uma das estatísticas anteriormente descritas. Os resultados do teste dependem do número de lags $\left(\Delta \mathbf{y}_{\mathbf{t}-\mathbf{i}}\right)$ incluídos no lado direito da eq. (3). Para escolha do número de lags, Enders (2004) propõe que sejam estimados modelos VAR de diferentes ordens para as variáveis em nível objeto do teste, e seja escolhido o número de defasagens igual à ordem do modelo com os menores valores para os critérios AIC e SBC.

\subsection{Teste de eficiência de curto prazo}

O processo convencional para testar a eficiência requer primeiramente o teste da presença de co-integração e, segundo, testar se o preço futuro no contrato comprado é um preditor não tendencioso do preço spot no vencimento do contrato. A regressão de co-integração é convencionalmente especificada como (KELLARD et al., 1999):

$$
s_{t}=\beta_{0}+\beta_{1} f_{t-1}+u_{t}
$$

onde $f_{t-1}$ refere-se ao preço futuro defasado do contrato comprado e 
$s_{t}$ refere-se ao preço spot verificado na data de vencimento do contrato futuro. A hipótese de não tendenciosidade diz respeito ao fato de $\beta_{0}=$ $0, \beta_{1}=1$ e $u_{t}$ é um ruído branco. Se $\beta_{1}=1$ não é rejeitado, estão não há evidência forte contra a hipótese que, no equilíbrio de longo-prazo, o preço spot é igual ao preço futuro acrescido de uma constante (possivelmente zero). Consequentemente pode-se especificar uma regressão de curto prazo relacionando a mudança no preço spot com a mudança na base $\left(f_{t-1}-s_{t-1}\right)$ e mudanças nos preços spot e futuro defasados:

$$
\Delta s_{t}=\alpha+\rho\left(f_{t-1}-s_{t-1}\right)+\sum_{i=1}^{k} \lambda_{i} \Delta s_{t-i}+\sum_{i=1}^{p} \gamma_{i} \Delta f_{t-i}+\varepsilon_{t}
$$

A equação acima proporciona um alicerce para testar se informação adicional para a base tem uso ótimo na previsão de mudanças no preço spot. Considera-se a significância estatística dos lags na equação acima como uma evidência de ineficiência.

Entretanto, se a eq. (7) for utilizada haverá problemas de autocorrelação serial nos resíduos, o que acaba por induzir o aparecimento de ineficiências em mercados em mercados que possivelmente são eficientes. Desta forma, optou-se por selecionar um horizonte de previsão de 28 dias. Assim, a nova equação pode ser reescrita como:

$$
s_{t}-s_{t-\tau}=\theta_{0}+\theta_{1}\left(f_{t-\tau}-s_{t-\tau}\right)+\sum_{i=1}^{k} \lambda_{i}\left(s_{t-i}-s_{(t-\tau)-i}\right)+\sum_{i=1}^{k} \gamma_{i}\left(f_{t-i}-f_{(t-\tau)-i}\right)+\varepsilon_{t}(8)
$$

onde $f_{t-\tau}$ é o preço futuro apurado 28 dias úteis antes do vencimento do contrato, $s_{t-\tau}$ é o preço spot apurado na mesma data que $f_{t-\tau}$.

A eq. (8) proporciona um método de previsão relevante para o preço spot. A variância do erro na previsão pode ser considerada, desta forma, a variância do erro. De outro lado, a eficiência do mercado futuro implicaria uma previsão de $f_{t-\tau}+E\left[\left(s_{t}-f_{t-\tau}\right)\right]$, permitindo a possibilidade de um desconto sistemático ou um prêmio no preço futuro. A variância do erro da predição pode ser estimada através de uma amostra da variância de $\left(s_{t}-f_{t-\tau}\right)$. A razão destas duas variâncias do erro de previsão proporciona uma estimativa da eficiência dos preços futuros como previsores do futuro preço spot. Desta forma, a medida de eficiência de curto-prazo pode ser escrita como: 


$$
\square_{c} \square \frac{(n \square 2 k \square 2)^{\square 1} \bigsqcup_{t \square 1}^{n} \square^{2}}{(n \square 1)^{\square 1} \square_{t \square 1}^{n}\left[\left(s_{t} \square f_{t \square}\right) \square\left(\overline{\left.s_{t} \square f_{t \square}\right)}\right]^{2}\right.}
$$

Onde n é o número de observações usadas na estimativa da eq. (8) e $(2 k+2)$ é o número de parâmetros estimados na equação. O numerador da eq. (9) é a variância do erro estimado na eq. (8), enquanto que o denominador é a variância da amostra do erro de previsão, baseado no preço futuro, corrigido pelos graus de liberdade. A obtenção de valor 1 na eq. (9) indicaria eficiência, ou seja, zero de ineficiência, e valores entre 0 e 1 indicariam variados graus de eficiência.

\section{Dados}

Para realização deste trabalho, foram utilizadas séries de preços diários referentes à cotação do último negócio dos contratos futuros de boi gordo e soja negociados na BM\&F. Para o boi gordo, os meses de vencimento foram de janeiro a novembro de 2005 e a série de preços futuros abrangeu oito meses antes do último dia de negociação do contrato, sendo excluídos os dias em que não houve negócios. A escolha desse intervalo de tempo deve-se à concentração de um maior número de negócios em um intervalo contínuo, isto é, sem que houvesse um grande número de dias sem negócios. Para o contrato futuro de soja da BM\&F os meses de vencimento escolhidos foram março, abril, maio, junho, julho, agosto, setembro e novembro de 2005. Já para o contrato de soja a granel da CBOT os meses de vencimento escolhidos foram janeiro, março, maio, julho, agosto, setembro e novembro de 2005 . O intervalo de abrangência dos dados teve variação entre sete e oito meses, baseando-se no mesmo critério do contrato futuro de boi gordo.

A série de preços diários do mercado spot de boi gordo foi disponibilizada pelo CEPEA/ESALQ/USP e refere-se aos preços em reais da arroba cotada em Campo Grande (MS). No caso da soja, os preços diários do mercado físico foram obtidos junto à Secretaria de Agricultura da Bahia e referem-se aos preços em reais da saca de $60 \mathrm{~kg}$ cotada em Barreiras (BA). As cotações de preços futuros foram obtidos junto à BM\&F e ao 
Barchart (2006) (para os preços futuros da CBOT). O período de abrangência foi similar ao da série de preços futuros. Em virtude da série de preços físicos da soja se encontrar em reais e os contratos futuros de soja negociados na BM\&F e na CBOT serem cotados em dólares norteamericanos (US\$), utilizou-se a taxa de cambio diária de reais por dólar para venda, disponibilizada pelo IPEA, para transformação dos preços no mercado físico para dólares por saca de $60 \mathrm{~kg}$.

\section{Resultados e discussões}

O primeiro passo consistiu em realizar os testes de raiz unitária nas variáveis pelo método de Dickey-Fuller Aumentado, conforme procedimento sequencial proposto por Enders (2004). Todas as estimações realizadas no trabalho foram realizadas por meio dos softwares Intercooled Stata 9.0 e Eviews 5.0. A Tabela 1 apresenta os resultados para os contratos futuros de boi gordo e soja. Para simplificar, foram reportados apenas os valores referentes à última etapa do teste de raiz unitária.

Pode-se verificar que as séries de preço spot e futuro referentes aos contratos de boi gordo com vencimento em janeiro, março, maio, junho, julho, setembro, outubro e novembro apresentaram ordens de integração diferentes, haja visto que uma das séries apresentou indícios de uma raiz unitária e a outra se mostrou estacionária. O mesmo ocorreu com os contratos de soja da BM\&F com vencimento em março, abril e maio. $\mathrm{O}$ fato das séries serem integradas de diferentes ordens impossibilitaria a realização do teste de co-integração entre estas séries. Apesar da rejeição da hipótese de existência de uma raiz unitária nestas séries ao nível de 5,0\% de significância, apenas na série de preços futuros referentes ao contrato de boi gordo com vencimento em junho pode-se rejeitar a hipótese de existência de uma raiz unitária ao nível de 1,0\%. Desta forma, considerou-se que, no caso do contrato de boi gordo, as séries de preços spot referentes aos contratos com vencimento em março, julho, outubro e novembro, e as séries de preços futuros referentes aos contratos com vencimento em janeiro e setembro são Integradas de ordem 1, ou I(1). Realizou-se o mesmo procedimento com os contratos de soja da BM\&F, especificamente com as séries de preços spot referentes aos contratos com vencimento em abril e maio, e também com a sé- 
rie de preços futuros referente ao contrato com vencimento em março. O teste de raiz unitária nas séries de preços spot e futuro referentes ao contrato de soja da CBOT indicaram que as mesmas são I(1).

Tabela 1 - Resultados do teste de raiz unitária nas séries de preços referentes aos contratos de boi gordo e soja da BM\&F

\begin{tabular}{|c|c|c|c|c|c|c|}
\hline \multirow[b]{2}{*}{ Commodity } & \multirow{2}{*}{$\begin{array}{l}\text { Contrato } \\
\text { (No. de } \\
\text { meses) }\end{array}$} & \multirow{2}{*}{$\begin{array}{l}\text { No. de } \\
\text { Obser- } \\
\text { vações }\end{array}$} & \multicolumn{2}{|c|}{ Modelo $1^{\mathrm{a}}$} & \multicolumn{2}{|c|}{ Modelo $2^{\text {b }}$} \\
\hline & & & $\begin{array}{c}\text { Spot } \\
\text { Estatística }\end{array}$ & $\begin{array}{c}\text { Futuro } \\
\text { Estatística }\end{array}$ & $\begin{array}{c}\text { Spot } \\
\text { Estatística }\end{array}$ & $\begin{array}{c}\text { Futuro } \\
\text { Estatística }\end{array}$ \\
\hline \multirow{11}{*}{ Boi gordo } & Jan 2005 (8) & 150 & $-1,69$ & $-2,41^{*}$ & $-7,27^{*}$ & $-7,18^{*}$ \\
\hline & Fev 2005 (8) & 163 & $-1,53$ & $-1,87$ & $-7,19^{*}$ & $-6,44^{*}$ \\
\hline & Mar 2005 (8) & 155 & $-2,19^{*}$ & $-1,88$ & $-7,06^{*}$ & $-6,60 *$ \\
\hline & Abr 2005 (8) & 162 & 1,41 & $-1,69$ & $-6,92 *$ & $-6,08^{*}$ \\
\hline & Mai 2005 (8) & 162 & $-1,85$ & $-2,01^{*}$ & $-6,66^{*}$ & $-6,98^{*}$ \\
\hline & Jun 2005 (8) & 161 & $-1,73$ & $-2,65^{*}$ & $-6,76^{*}$ & $-7,22^{*}$ \\
\hline & Jul 2005 (8) & 164 & $-2,57^{*}$ & $-1,19$ & $-7,30^{*}$ & $-7,49^{*}$ \\
\hline & Ago 2005 (8) & 168 & $-2,07^{*}$ & $-2,79 *$ & $4,34^{*}$ & $2,10^{*}$ \\
\hline & Set 2005 (8) & 167 & $-0,29$ & $-1,91^{*}$ & $-6,54^{*}$ & $-9,26^{*}$ \\
\hline & Out 2005 (8) & 165 & $-2,99 *$ & $-1,05$ & $-8,21^{*}$ & $-7,27^{*}$ \\
\hline & Nov 2005 (8) & 161 & $-3,29^{*}$ & $-1,04$ & $-7,70^{*}$ & $-7,15^{*}$ \\
\hline \multirow{8}{*}{$\begin{array}{l}\text { Soja } \\
\text { BM\&F }\end{array}$} & Mar 2005 (7) & 38 & $-0,38$ & $-3,56^{*}$ & $-5,60^{*}$ & $-4,16^{*}$ \\
\hline & Abr 2005 (8) & 90 & $-2,91^{*}$ & 1,15 & $-7,39^{*}$ & $-7,10^{*}$ \\
\hline & Mai 2005 (8) & 145 & $-3,12^{*}$ & 0,17 & $-9,69^{*}$ & $-7,38^{*}$ \\
\hline & Jun 2005 (7) & 65 & $-0,62$ & 0,50 & $-5,94^{*}$ & $-4,97^{*}$ \\
\hline & Jul 2005 (7) & 84 & $-0,23$ & 1,08 & $-6,53^{*}$ & $-7,94^{*}$ \\
\hline & Ago 2005 (7) & 110 & 0,13 & 0,98 & $-7,15^{*}$ & $-8,92^{*}$ \\
\hline & Set $2005(7)$ & 119 & $-3,05^{*}$ & $-3,31^{*}$ & $-9,29 *$ & $-6,76^{*}$ \\
\hline & Nov 2005 (7) & 107 & $-0,54$ & 0,01 & $-6,56^{*}$ & $-6,70^{*}$ \\
\hline \multirow{7}{*}{$\begin{array}{l}\text { Soja } \\
\text { CBOT }\end{array}$} & Jan 2005 (8) & 189 & $-1,48$ & $-1,89$ & $-13,70^{*}$ & $-11,61^{*}$ \\
\hline & Mar 2005 (8) & 147 & $-0,79$ & $-0,05$ & $-13,12^{*}$ & $-11,88^{*}$ \\
\hline & Mai 2005 (8) & 164 & $-1,64$ & $-0,66$ & $-13,25^{*}$ & $-6,00^{*}$ \\
\hline & Jul 2005 (8) & 163 & $-0,75$ & 0,34 & $-16,01^{*}$ & $-6,53^{*}$ \\
\hline & Ago 2005 (8) & 166 & $-0,39$ & 0,03 & $-14,91^{*}$ & $-7,37^{*}$ \\
\hline & Set 2005 (8) & 166 & $-0,89$ & $-0,40$ & $-16,00^{*}$ & $-6,48^{*}$ \\
\hline & Nov 2005 (8) & 171 & $-0,93$ & $-0,90$ & $-16,51^{*}$ & $-7,22 *$ \\
\hline
\end{tabular}

Fonte: dados da pesquisa.

Nota: $\left({ }^{*}\right)$ Significativo ao nível de 5,0\%. Valores críticos de Fuller (1976).

(a) Modelo 1: $\Delta y_{t}=a_{0}+\gamma y_{t-1}+a_{2} t+\sum_{i=1}^{k} \beta_{i} \Delta y_{t-i}+e_{t}$, nas versões ( $i$ ) com constante e tendência, (ii) com constante e sem tendência. (iii) sem constante e sem tendência;

(b) Modelo 2: $\Delta\left(\Delta y_{t}\right)=\gamma \Delta y_{t-1}+\sum_{i=1}^{k-1} \beta_{i} \Delta\left(\Delta y_{t-i}\right)+e_{t}$, definido depois de constada a inexistência de termos deterministas e para verificar a estacionariedade das séries.

RER, Rio de Janeiro, vol. 46, oㅡ 01, p. 075-096, jan/mar 2008 - Impressa em abril 2008 
Os resultados dos testes de co-integração entre os preços spot e futuro da BM\&F para o boi gordo e a soja são apresentados nas Tabelas 2 e 3, respectivamente, ao passo que na Tabela 4 constam os resultados dos testes de co-integração entre os preços spot e futuro do contrato de soja da CBOT. Em virtude das séries de preços spot e futuro do contrato de boi gordo com vencimento em junho serem integradas de ordens diferentes, o teste de co-integração não foi realizados entre as mesmas. A escolha dos lags ótimos para cada série foi realizada através do cálculo do SBC, o qual tende a escolher especificações mais simples. A coluna " $\mathrm{H}_{0}=r$ " indica a hipótese que está sendo testada (a existência de 0 vetor de co-integração ou a existência de 1 vetor de co-integração). A coluna intitulada "Traço" refere-se ao valor do teste do Traço, cujo cálculo é apresentado na seção 3.1 deste trabalho. A coluna "Dec." indica a decisão: $\mathrm{R}$ significa que rejeita-se a hipótese nula de que o número de vetores de co-integração seja igual a $r$ (apresentado na coluna " $\mathrm{H}_{0}$ $=r$ "), ao nível de 5,0\% de significância; NR indica que não se pode rejeitar a hipótese nula ao nível de 5,0\% de significância (a ausência de decisão em algumas colunas com $\mathrm{H}_{0}: r=1$ refere-se ao fato de que $\mathrm{H}_{0}: r=0$ não pode ser rejeitado, e, portanto, a existência de 1 vetor é automaticamente rejeitada).

No caso do boi gordo, os testes sem tendência linear indicaram co-integração entre os preços no mercado spot e os preços no mercado futuro para os contratos com vencimento em março, abril, outubro e novembro de 2005. Nos testes com tendência linear detectou-se co-integração nos contratos com vencimento em abril e setembro de 2005. Em se tratando do contrato de soja da BM\&F, evidências de cointegração foram encontradas, nos testes sem tendência linear, para os contratos com vencimento em abril, maio e novembro de 2005 . O contrato de soja da CBOT apresentou co-integração nos contratos com vencimento em janeiro, março e novembro de 2005 nos testes sem tendência linear, e também em setembro de 2005 nos testes com tendência linear. A evidência da presença de co-integração indica uma relação de equilíbrio de longo-prazo entre os dois preços (spot e futuro), em que diferenças nas trajetórias dos mesmos seriam motivadas apenas por choques de curto-prazo. 
Tabela 2 - Testes de co-integração entre preços spot e preços futuros para os contratos de boi gordo

\begin{tabular}{|c|c|c|c|c|c|c|c|c|}
\hline \multirow[b]{2}{*}{$\begin{array}{l}\text { No. } \\
\text { lags }\end{array}$} & \multirow[b]{2}{*}{ Contrato } & \multirow[b]{2}{*}{$\begin{array}{l}\mathrm{H}_{0} \\
=r\end{array}$} & \multicolumn{3}{|c|}{ Sem tendência linear } & \multicolumn{3}{|c|}{ Com tendência linear } \\
\hline & & & Traço & $\begin{array}{c}\text { Val. } \\
\text { críticos a } \\
5,0 \%\end{array}$ & Dec. & Traço & $\begin{array}{c}\text { Val. } \\
\text { críticos a } \\
5,0 \%\end{array}$ & Dec. \\
\hline \multirow{2}{*}{1} & \multirow{2}{*}{ Jan 05} & 0 & 4,5602 & 15,41 & NR & 4,0232 & 18,17 & NR \\
\hline & & 1 & 0,7295 & 3,76 & & 0,0093 & 3,74 & \\
\hline \multirow{2}{*}{2} & \multirow{2}{*}{ Fev 05} & 0 & 11,2828 & 15,41 & NR & 7,5805 & 18,17 & NR \\
\hline & & 1 & 0,3415 & 3,76 & & 0,9947 & 3,74 & \\
\hline \multirow{2}{*}{1} & \multirow{2}{*}{ Mar 05} & 0 & 16,6058 & 15,41 & $\mathrm{R}$ & 14,0463 & 18,17 & NR \\
\hline & & 1 & 0,1017 & 3,76 & NR & 1,6117 & 3,74 & \\
\hline \multirow{2}{*}{1} & \multirow{2}{*}{ Abr 05} & 0 & 16,4446 & 15,41 & $\mathrm{R}$ & 18,8081 & 18,17 & $\mathrm{R}$ \\
\hline & & 1 & 0,2062 & 3,76 & NR & 3,0787 & 3,74 & NR \\
\hline \multirow{2}{*}{1} & \multirow{2}{*}{ Mai 05} & 0 & 12,5295 & 15,41 & NR & 17,0161 & 18,17 & NR \\
\hline & & 1 & 0,0961 & 3,76 & & 3,8927 & 3,74 & \\
\hline \multirow{2}{*}{2} & \multirow{2}{*}{ Jul 05} & 0 & 9,9917 & 15,41 & NR & 15,1973 & 18,17 & NR \\
\hline & & 1 & 0,6824 & 3,76 & & 4,3929 & 3,74 & \\
\hline \multirow{2}{*}{1} & \multirow{2}{*}{ Ago 05} & 0 & 6,5429 & 15,41 & NR & 12,5003 & 18,17 & NR \\
\hline & & 1 & 0,0151 & 3,76 & & 4,4695 & 3,74 & \\
\hline \multirow{2}{*}{2} & \multirow{2}{*}{ Set 05} & 0 & 10,9865 & 15,41 & NR & 17,0778 & 18,17 & $\mathrm{R}$ \\
\hline & & 1 & 2,7667 & 3,76 & & 0,0085 & 3,74 & NR \\
\hline \multirow{2}{*}{2} & \multirow{2}{*}{ Out 05} & 0 & 17,8551 & 15,41 & $\mathrm{R}$ & 23,9103 & 18,17 & $\mathrm{R}$ \\
\hline & & 1 & 1,0577 & 3,76 & NR & 7,0302 & 3,74 & $\mathrm{R}$ \\
\hline \multirow{2}{*}{4} & \multirow[t]{2}{*}{ Nov 05} & 0 & 18,5582 & 15,41 & $\mathrm{R}$ & 25,1517 & 18,17 & $\mathrm{R}$ \\
\hline & & 1 & 1,7901 & 3,76 & NR & 6,3687 & 3,74 & $\mathrm{R}$ \\
\hline
\end{tabular}

Fonte: dados da pesquisa. 
Tabela 3 - Testes de co-integração entre preços spot e preços futuros dos contratos de soja da BM\&F

\begin{tabular}{|c|c|c|c|c|c|c|c|c|}
\hline \multirow[b]{2}{*}{$\begin{array}{l}\text { No. } \\
\text { lags }\end{array}$} & \multirow[b]{2}{*}{ Contrato } & \multirow[b]{2}{*}{$\begin{array}{l}\mathrm{H}_{0} \\
=r\end{array}$} & \multicolumn{3}{|c|}{ Sem tendência linear } & \multicolumn{3}{|c|}{ Com tendência linear } \\
\hline & & & Traço & $\begin{array}{c}\text { Val. } \\
\text { críticos a } \\
5,0 \%\end{array}$ & Dec. & Traço & $\begin{array}{c}\text { Val. } \\
\text { críticos a } \\
5,0 \%\end{array}$ & Dec. \\
\hline \multirow[b]{2}{*}{1} & \multirow{2}{*}{ Mar 05} & 0 & 27,7642 & 15,41 & $\mathrm{R}$ & 26,8811 & 18,17 & NR \\
\hline & & 1 & 10,9615 & 3,76 & $\mathrm{R}$ & 10,1602 & 3,74 & \\
\hline \multirow{2}{*}{1} & \multirow{2}{*}{ Abr 05} & 0 & 19,5013 & 15,41 & $\mathrm{R}$ & 13,1961 & 18,17 & NR \\
\hline & & 1 & 0,9229 & 3,76 & NR & 0,0299 & 3,74 & \\
\hline \multirow{2}{*}{1} & \multirow{2}{*}{ Mai 05} & 0 & 24,7977 & 15,41 & $\mathrm{R}$ & 28,4094 & 18,17 & $\mathrm{R}$ \\
\hline & & 1 & 0,7961 & 3,76 & NR & 5,7191 & 3,74 & $\mathrm{R}$ \\
\hline \multirow{2}{*}{1} & \multirow{2}{*}{ Jun 05} & 0 & 8,6158 & 15,41 & NR & 10,6873 & 18,17 & NR \\
\hline & & 1 & 2,4937 & 3,76 & & 2,9390 & 3,74 & \\
\hline \multirow{2}{*}{1} & \multirow{2}{*}{ Jul 05} & 0 & 15,0317 & 15,41 & NR & 17,9949 & 18,17 & NR \\
\hline & & 1 & 2,7211 & 3,76 & & 3,0236 & 3,74 & \\
\hline \multirow{2}{*}{1} & \multirow{2}{*}{ Ago 05} & 0 & 15,2746 & 15,41 & NR & 17,4243 & 18,17 & NR \\
\hline & & 1 & 3,4443 & 3,76 & & 5,5638 & 3,74 & \\
\hline \multirow{2}{*}{1} & \multirow{2}{*}{ Set 05} & 0 & 31,6398 & 15,41 & $\mathrm{R}$ & 29,4445 & 18,17 & $\mathrm{R}$ \\
\hline & & 1 & 13,9465 & 3,76 & $\mathrm{R}$ & 10,4403 & 3,74 & $\mathrm{R}$ \\
\hline \multirow{2}{*}{1} & \multirow{2}{*}{ Nov 05} & 0 & 31,0509 & 15,41 & $\mathrm{R}$ & 35,4182 & 18,17 & $\mathrm{R}$ \\
\hline & & 1 & 3,4347 & 3,76 & NR & 5,5706 & 3,74 & $\mathrm{R}$ \\
\hline
\end{tabular}

Fonte: dados da pesquisa.

Tabela 4 - Testes de co-integração entre preços spot e preços futuros para os contratos de soja da CBOT

\begin{tabular}{|c|c|c|c|c|c|c|c|c|}
\hline \multirow[b]{2}{*}{$\begin{array}{l}\text { No. } \\
\text { lags }\end{array}$} & \multirow[b]{2}{*}{ Contrato } & \multirow[b]{2}{*}{$\begin{array}{l}\mathrm{H}_{0} \\
=r\end{array}$} & \multicolumn{3}{|c|}{ Sem tendência linear } & \multicolumn{3}{|c|}{ Com tendência linear } \\
\hline & & & Traço & $\begin{array}{c}\text { Val. } \\
\text { críticos a } \\
5,0 \%\end{array}$ & Dec. & Traço & $\begin{array}{c}\text { Val. } \\
\text { críticos a } \\
5,0 \%\end{array}$ & Dec. \\
\hline \multirow{2}{*}{1} & \multirow{2}{*}{ Jan 05} & 0 & 19,2533 & 15,41 & $\mathrm{R}$ & 21,6470 & 18,17 & $\mathrm{R}$ \\
\hline & & 1 & 0,9738 & 3,76 & NR & 2,8561 & 3,74 & NR \\
\hline \multirow{2}{*}{1} & \multirow{2}{*}{ Mar 05} & 0 & 22,5684 & 15,41 & $\mathrm{R}$ & 18,2152 & 18,17 & $\mathrm{R}$ \\
\hline & & 1 & 2,2463 & 3,76 & NR & 0,1906 & 3,74 & NR \\
\hline \multirow{2}{*}{1} & \multirow{2}{*}{ Mai 05} & 0 & 14,8181 & 15,41 & NR & 24,2128 & 18,17 & NR \\
\hline & & 1 & 2,5586 & 3,76 & & 9,4258 & 3,74 & \\
\hline \multirow{2}{*}{1} & \multirow{2}{*}{ Jul 05} & 0 & 14,6429 & 15,41 & NR & 28,0570 & 18,17 & $\mathrm{R}$ \\
\hline & & 1 & 4,3387 & 3,76 & & 5,8309 & 3,74 & $\mathrm{R}$ \\
\hline \multirow{2}{*}{1} & \multirow{2}{*}{ Ago 05} & 0 & 12,8107 & 15,41 & NR & 25,3111 & 18,17 & $\mathrm{R}$ \\
\hline & & 1 & 4,7000 & 3,76 & & 4,9687 & 3,74 & $\mathrm{R}$ \\
\hline \multirow{2}{*}{1} & \multirow{2}{*}{ Set 05} & 0 & 10,3647 & 15,41 & NR & 27,9059 & 18,17 & $\mathrm{R}$ \\
\hline & & 1 & 2,3437 & 3,76 & & 3,7935 & 3,74 & $\mathrm{R}$ \\
\hline \multirow{2}{*}{1} & \multirow{2}{*}{ Nov 05} & 0 & 16,8850 & 15,41 & $\mathrm{R}$ & 28,4679 & 18,17 & $\mathrm{R}$ \\
\hline & & 1 & 0,8884 & 3,76 & NR & 6,9666 & 3,74 & $\mathrm{R}$ \\
\hline
\end{tabular}

Fonte: dados da pesquisa. 
Em virtude da disponibilidade de dados, o teste de eficiência de curto-prazo foi realizado apenas para o contrato futuro de boi gordo da BM\&F. Estimou-se inicialmente a eq. (8) com 10 lags $(k=10)$, sendo excluídos os que se mostravam não significantes a 10,0\%, preservando a simetria entre $\left(s_{t-i}-s_{(t-\tau)-i}\right)$ e $\left(f_{t-i}-f_{(t-\tau)-i}\right)$. Este procedimento remove toda a evidência de correlação serial. Os coeficientes estimados são apresentados na Tabela 5, sendo que $n$ representa o número de observações utilizadas na estimação da equação, $F$ representa o teste para a hipótese nula de que todos os coeficientes da equação sejam iguais a zero e DW representa o teste de Durbin-Watson para correlação serial.

Tabela 5 - Resultado da estimação da eq. (8) para o teste de eficiência de curto-prazo do mercado futuro de boi gordo

\begin{tabular}{cc}
\hline Coeficientes & $\begin{array}{c}\text { Valor dos coeficientes } \\
\text { (estatística- } t \text { ) }\end{array}$ \\
\hline$\theta_{0}$ & $-0,6351202(-0,85)$ \\
$\theta_{1}$ & $0,2674346(1,10)$ \\
$\lambda_{1}$ & $0,7100015(3,40)$ \\
$\lambda_{2}$ & $-0,0160045(-0,07)$ \\
$\lambda_{3}$ & $-0,3560741(-1,69)$ \\
$\gamma_{1}$ & $-0,5474652(-2,18)$ \\
$\gamma_{2}$ & $0,2122519(0,78)$ \\
$\gamma_{3}$ & $0,451542(1,82)$ \\
& $\mathrm{n}=51$ \\
& $\mathrm{~F}(7,43)=3,70$ \\
$\mathrm{DW}=1,9885$
\end{tabular}

Fonte: dados da pesquisa.

A presença de lags significativos na estimação da eq. (8) mostra evidências de alguma ineficiência de curto-prazo. Utilizando os dados da equação estimada, efetuou-se o cálculo do indicador de eficiência do mercado futuro de boi gordo através da eq. (9). O valor de $\phi_{C}$ foi de 0,6973 de eficiência, numa escala de 0 (totalmente ineficiente) até 1 (totalmente eficiente). Este valor apresenta-se elevado, em comparação com o cálculo de Kellard et at., (1999) para a eficiência do contrato 
futuro de boi gordo da CBOT. Isto indica que o mercado futuro de boi gordo da BM\&F possui capacidade satisfatória de auxiliar o produtor na descoberta de preço.

\section{Conclusões}

Este trabalho teve por objetivo avaliar se existe uma ligação estatística de longo-prazo entre os mercados físico (spot) e futuro de boi gordo e soja, de modo que o produtor possa utilizar este último mercado como forma de auxiliá-lo no processo de descoberta do preço spot futuro.

Em se tratando do boi gordo, evidências de ligação entre os mercados spot e futuro, detectadas por meio da técnica de co-integração, foram obtidas para os contratos futuros com vencimento em março, abril, setembro, outubro e novembro/2005, ou seja, $45,5 \%$ dos contratos futuros de boi gordo analisados. Nos meses onde há concentração da venda de boi gordo, setembro e outubro, e, consequentemente, há uma tendência de maior demanda por redução do risco de preço, foram detectadas evidências de co-integração, indicando que os contratos futuro nestes vencimentos podem auxiliar os produtores de maneira eficiente no processo de descoberta de preço. A existência de co-integração no caso do boi gordo contrasta com os resultados de alguns trabalhos, como Bessler \& Covey (1991), Fortenbery \& Zapata (1993) e Covey \& Bessler (1995), os quais não encontram co-integração entre os preços spot e futuros para commodities não-estocáveis (entre as quais o boi gordo) e também argumentavam não ser possível esperar tal tipo de evidência. Por outro lado, tais resultados se aproximam do trabalho de Yang et al. (2001) que encontrou evidências de co-integração entre mercado spot e futuro para boi gordo, porco vivo e bezerro nos EUA.

No caso do contrato de soja da BM\&F, a ligação entre o mercado spot e futuro foi detectada para os contratos futuros com vencimento em abril, maio e novembro de 2005 , o que representa $37,5 \%$ dos contratos de soja analisados, sendo que os meses de abril e maio coincidem com o período de safra, demonstrando que os contratos com estes vencimentos podem prover melhores informações aos produtores, em relação aos contratos com outros vencimentos, na prospecção do preço futuro no mercado spot. Para o contrato de soja da CBOT, detectou-se 
co-integração nos contratos com vencimento em janeiro, março, setembro e novembro/2005. Estas evidências também estão em conformidade com Yang et al., (2001), que encontrou evidências de co-integração entre o mercado físico e futuro da soja nos EUA.

$O$ indicador de eficiência representado por $\phi_{C}$ mostra que o contrato futuro de boi gordo da BM\&F possui um nível relevante de eficiência, de acordo com sua grande liquidez, o que demonstra sua performance adequada para descoberta do preço spot futuro por parte do produtor.

A detecção da existência de co-integração entre os preços spot e futuro das commodities boi gordo e soja pode estar relacionado às características institucionais destes mercados. No caso do boi gordo, há uma baixa presença de integração contratual e vertical. Os frigoríficos se abastecem diretamente do mercado spot de animais, em geral adquirindo o produto de intermediários especializados. Desta forma, o pecuarista tem que procurar assegurar o preço de venda através do mercado futuro. Como as expectativas de preços são formadas por um número maior de agentes, o preço futuro tende a acompanhar o preço spot. No caso da soja, grande parte dos produtores asseguram o preço de seu produto através das tradings, que adquirem antecipadamente a produção ou recebem a mesma como pagamento por insumos. Apesar de muitas tradings utilizarem mercados futuros de soja com maior liquidez, como a CBTO, por exemplo, o número de contratos futuros de soja na BM\&F apresentou elevação expressiva em 2005, o que pode ter contribuído para a detecção de co-integração em alguns contratos. Por fim, o contrato futuro de soja da CBOT é utilizado por produtores e tradings norte-americanas e também da América do Sul e demais continentes, havendo, portanto, liquidez em contratos com diferentes meses de vencimento, e não apenas naqueles com vencimento durante a safra de grãos dos EUA, o qual pode ter facilitado a detecção da co-integração.

A explicação pela ausência de co-integração nos demais contratos não foi objeto de estudo neste trabalho, mas cumpre-se levantar algumas hipóteses que podem ser testadas em trabalhos posteriores. No caso do boi gordo, o número de observações utilizadas nos testes não varia consideravelmente de um contrato para outro, o que possibilita, em princípio, descartarmos tal fator como influenciador dos resultados dos testes de co-integração nesta commodity. Em se tratando da soja, 
cujo contrato futuro da BM\&F possui menor liquidez e, consequentemente, um menor número de observações em determinados meses, a co-integração parece também não ter sido afetada por esta variação no tamanho das amostras, pois a mesma foi detectada em contratos com diferentes números de observações (abril, maio e novembro).

Outra hipótese pode estar relacionada à disponibilidade de informações relativas ao mercado físico destas commodities. O período de maior quantidade de vendas de boi gordo (setembro e outubro) e de safra da soja (abril e maio) são os períodos em que os produtores naturalmente aguardam que seus produtos estejam acabados para venda e, desta forma, meses antes deste período os mesmo demandam informações sobre como estará o mercado naquele ponto futuro. $\mathrm{O}$ aumento da demanda impulsiona uma maior oferta de informações, tanto públicas quanto privadas, elaborando previsões relativas à oferta, preço, taxa de câmbio, valor do frete, entre outras, que estarão vigentes no período de maior produção (ou safra). O mercado futuro com contrato de vencimento nestes meses agrega todas essas informações e, desta forma, o seu processo de descoberta de preço torna-se mais eficiente nestes meses, o qual foi detectado pela co-integração.

As limitações deste trabalho devem-se à indisponibilidade de outras séries de preços spot cotados em outras localidades, pois características influenciando a demanda e oferta nos locais de formação dos preços spot podem influenciar os resultados finais obtidos. Repetindo a análise para preços cotados em diferentes locais propiciaria evidências mais robustas da existência ou não de integração entre os mercados futuro e spot do boi gordo e da soja.

Estudos futuros podem ser realizados no sentido de comparar a eficiência no contrato futuro de soja da CBOT e da BM\&F, os quais não puderam ser realizados devido à indisponibilidade de preços spot e futuros, respectivamente.

\section{Referências bibliográficas}

BARCHART. Advanced commodities. Disponível em: < http://www. barchart.com/info/futures_prices.html > . Acesso em: 10 jan. 2006. 
BESSLER, D.A.; COVEY, T. Cointegration: some results on U.S. cattle prices. Journal of Futures Markets, v. 11, n. 4, p. 461-474, Aug.1991. BOLSA DE MERCADORIAS \& FUTUROS - BM\&F. Relatório agropecuário mensal - novembro de 2005. 2005a. Disponível em: < http:// www.bmf.com.br/2004/pages/

imprensa1/relatorios/RelMensal/RelatorioMensal_Novembro2005. zip > . Acesso em: 06 jan. 2006.

BOLSA DE MERCADORIAS \& FUTUROS - BM\&F. Especificações do contrato futuro de boi gordo denominado em reais. 2005b. Disponível em: < http://www.bmf.com.br/2004/pages/contratos1/Agropecuarios/ContratosAgroBoiReais1.asp > . Acesso em: 06 jan. 2006.

BOLSA DE MERCADORIAS \& FUTUROS - BM\&F. Especificações do contrato futuro de soja em grão a granel. 2005c. Disponível em: < http:// www.bmf.com.br/2004/pages/

contratos1/Agropecuarios/ContratosAgroBoiReais1.asp > . Acesso em: 06 jan. 2006.

CHICADO BOARD OF TRADE - CBOT. Monthly statistics: December 2005. Disponível em: < http://www.cbot.com/

cbot/docs/66627.pdf > . Acesso em: 10 jan. 2006.

COMPANHIA NACIONAL DE ABASTECIMENTO - CONAB. Soja - Brasil. 2005. Disponível em: < http://www.conab.gov.br/download/safra/ SojaSerieHist.xls > . Acesso em: 06 jan. 2006.

COVEY, T.; BESSLER, D.A. Asset storability and the information content of inter-temporal prices. Journal of Empirical Finance, v.2, n. 2, p. 103-115, June 1995.

DICKEY, D.; FULLER, W. Likelihood ratio statistics for autoregressive time series with a unit root. Econometrica, v. 49, n. 4, p. 1057-1072, July 1981.

ENDERS, W. Applied econometric time series. New York: John Wiley \& Sons, 2004. 466 p.

ENGLE, R.J.; GRANGER, C.W.J. Co-integration and error correction: 
representation, estimation and testing. Econometrica, v. 55, n. 2, p. 251-276, Mar. 1987.

FNP CONSULTORIA \& AGROINFORMATIVOS. Agrianual 2005. FNP Consultoria \& Agroinformativos: São Paulo, 2004. 500 p.

FORTENBERY, T.R.; ZAPATA, H. O. An examination of cointegration relations between futures and local grain markets. Journal of Futures Markets, v. 13, n. 8, p. 921-932, Dec. 1993.

FULLER, W.A. Introduction to statistical time series. New York: John Wiley \& Sons, 1976. 480 p.

INSTITUTO FNP. Anualpec 2005. São Paulo: Instituto FNP, 2005. 340 p. JOHANSEN, S. Statistical analysis of cointegration factors. Journal of Economic Dynamic and Control, v. 12, n. 2-3, p. 231-254, Sept. 1988.

JOHANSEN, S.; JUSELIUS, K. Maximum likelihood estimation and inference on cointegration - with application on the demand for money. Oxford Bulletin of Economics \& Statistics, v. 52, n. 2, p. 169-210, May 1990.

KELLARD, N.; NEWBOLD, P.; RAYNER, T.; ENNEW, C. The relative efficiency of commodity futures markets. The Journal of Futures Markets, v. 19, n. 4, p. 413-432, June 1999.

LAI, K.S., LAI, M.. A cointegration test for market efficiency. The Journal of Futures Markets, v. 11, n. 5, p. 567-575, Oct. 1991.

LJUNG, G.; BOX, G. On a measure of lack of fit in time series models. Biometrika, v. 65, n. 2, p. 297-303, Aug. 1978.

MACKINNON, J.G.; HAUG, A.A.; MICHELIS, L. Numerical distribution functions of likelihood ratio tests for cointegration. Journal of Applied Econometrics, v. 14, n. 5, p. 563-577, Sept./Oct. 1999.

SARTORIS, A. Estatística e introdução à econometria. São Paulo: Saraiva, 2003. $426 \mathrm{p}$.

STOCK, J.; WATSON, M. Testing for common trends. Journal of the American Statistical Association, v. 83, n. 404, p. 1097-1107, Dec. 1988. 
WANG, G.H.K.; YAU, J. A time series approach to testing for market linkage: unit root and cointegration tests. The Journal of Futures Markets, v. 14, n. 4, p. 457-474, June 1994.

YANG, J.; BESSLER, D.A.; LEATHAM, D.J. Asset storability and price discovery in commodity futures markets: a new look. The Journal of Futures Markets, v. 21, n. 3, p. 279-300, Mar. 2001. 